\title{
FORRAJE VERDE HIDROPÓNICO Y ORGANOPÓNICO DE MAÍZ COMO SUPLEMENTO NUTRICIONAL PARA OVINOS DEL PIEDEMONTE AMAZÓNICO
}

\author{
HYDROPONIC AND ORGANOPONIC GREEN FORAGE OF CORN AS A \\ NUTRITIONAL SUPPLEMENT FOR SHEEP IN THE AMAZON FOOTHILLS
}

\author{
Juan Pablo Narváez Herrera • jpnarvaez84@misena.edu.co \\ Zootecnista, Especialista en Nutrición Animal Sostenible, Magister en Ciencias Agrarias, Centro Agroforestal y \\ Acuícola Arapaima, SENA Regional Putumayo, Puerto Asís, Putumayo Colombia.
}

\section{Elizabeth Marcela Guerrero Guerrero•marcelitag2@yahoo.es Ingeniera Agrónoma, Especialista en Gestión Pública, Magister en Ciencias Agrarias, Centro Agroforestal y Acuícola Arapaima, SENA Regional Putumayo, Puerto Asís, Putumayo Colombia.}

Citación: Narváez-Herrera, J. P., Guerrero-Guerrero, E.M. (2022). Forraje verde hidropónico y organopónico de maíz como suplemento nutricional para ovinos del piedemonte amazónico. Revista de Investigación Agraria y Ambiental, 13(1), 253 - 266. DOI: https://doi.org/10.22490/21456453.4535

\section{RESUMEN}

Contextualización: Los rumiantes tienen una ventaja comparativa con relación a otras especies, gracias a la capacidad de digerir las fracciones de la fibra presente en los forrajes.

Vacío de conocimiento: En regiones tropicales, debido a las condiciones medioambientales cambiantes, se hace más difícil la producción de pasturas de alta calidad nutricional, derivando así en una estacionalidad forrajera. Esto conlleva a que sea necesaria la suplementación con alimentos comerciales para los animales, lo que incrementa los costos de producción.

Propósito del estudio: Evaluar el efecto de la suplementación con forraje verde hidropónico (FVH) y forraje verde organopónico (FVO) de maíz sobre el rendimiento productivo de ovinos en fase de levante, en el piedemonte amazónico del Departamento del Putumayo.

Metodología: Se utilizaron 12 corderos Dorper enteros con edad de 90 días y peso $14.62 \mathrm{~kg}$, los cuales se distribuyeron para la aplicación de tres tratamientos consistentes en: T0: dieta base (DB), T1: forraje verde hidropónico (FVH) y T2: forraje verde organopónico (FVO). Se evaluó la ganancia de peso total (GPT), ganancia diaria de peso
(GDP), consumo total de alimento (CTA), conversión alimenticia (CA) y eficiencia alimenticia (EA) durante un periodo de suplementación de 45 días. Se utilizó un diseño completamente al azar. Los datos se analizaron mediante análisis de varianza y las medias fueron separadas mediante la prueba de Tukey con un nivel de significancia de $(p<0.05)$.

Resultados y conclusiones: Se encontraron diferencias $(p<0.05)$ en la GPT $3.66 \mathrm{~kg}$ (T1), $3.61 \mathrm{~kg}$ (T2) y $2.35 \mathrm{~kg}$ (T0); de igual manera para la GDP $81.39 \mathrm{~g}$ (T1), $80.30 \mathrm{~g}$ (T2) y $52.22 \mathrm{~g}$ (T0); para el CTA 1.33 $\mathrm{kg}(\mathrm{T} 1), 1.27 \mathrm{~kg}(\mathrm{~T} 2)$ y $1.05 \mathrm{~kg}(\mathrm{~T} 0)$; la CA se estimó para el T1 en 3.23, 3.27 en T2 y 5.13 en T0; y la EA fue de 0.063 (T1), 0.061 (T2) y 0.050 (T0), evidenciando la influencia del tipo de dieta utilizada. La implementación de FVH y FVO se convierte en una alternativa viable de suplementación en los sistemas de producción de ovinos, en los que las condiciones medioambientales hacen difícil el cultivo de forrajes para pastoreo. Se concluye que la inclusión del 30 \% de FVH de maíz en la dieta de ovinos en crecimiento mejora su rendimiento productivo.

Palabras Clave: forraje; nutrición; rumiantes; suplementación 


\section{ABSTRACT}

Contextualization: Ruminants have a comparative advantage over other species, because their ability to digest the fiber fractions present in forages.

Knowledge gap: In tropical regions, due to changing environmental conditions, it is more difficult to produce pastures of high nutritional quality, resulting in forage seasonality that leads to supplementation with commercial feedstuffs that increase production costs.

Purpose: Evaluate the effect of supplementation with hydroponic green fodder (HGF) and organoponic green fodder (OGF) of corn on the productive performance of sheeps during the rearing phase, in the Amazonian piedmont of the Department of Putumayo.

Methodology: Twelve whole Dorper lambs, aged 90 days and weighing 14.62 $\mathrm{kg}$, were used. They were distributed to three treatments consisting of: TO: base diet (BD), T1: hydroponic green fodder (HGF), T2: organoponic green fodder (OGF). Total weight gain (TWG), daily weight gain (DWG), total feed intake (TFI), feed conversion (FC) and feed efficiency (FE) were evaluated during a supplementation period of 45 days. A completely randomized design was used. Data was analyzed by analysis of variance and means were separated by Tukey's test with a significance level of $(p<0.05)$.

Results and conclusions: Differences $(p<0.05)$ were found in TWG $3.66 \mathrm{~kg}(\mathrm{~T} 1)$, $3.61 \mathrm{~kg}$ (T2) and $2.35 \mathrm{~kg}$ (T0); similarly, to DWG $81.39 \mathrm{~g}(\mathrm{~T} 1), 80.30 \mathrm{~g}(\mathrm{~T} 2)$ and 52.22 g (T0); TFI $1.33 \mathrm{~kg}(\mathrm{~T} 1), 1.27 \mathrm{~kg}$ (T2) and $1.05 \mathrm{~kg}$ (T0); the FC was estimated for T1 at $3.23,3.27$ in $\mathrm{T} 2$ and 5.13 in T0; and the FE was 0.063 (T1), 0.061 (T2) and 0.050 (T0), evidencing the influence of the type of diet used. The implementation of HGF and OGF becomes a viable alternative for food supplementation in sheeps production systems, where environmental conditions make it difficult to cultivate forage for grazing. It was concluded that the inclusion of $30 \%$ of corn HGF in the diet of growing sheep improves their productive performance.

\footnotetext{
Keywords: forage; nutrition; ruminants;
} 
RESUMEN GRÁFICO
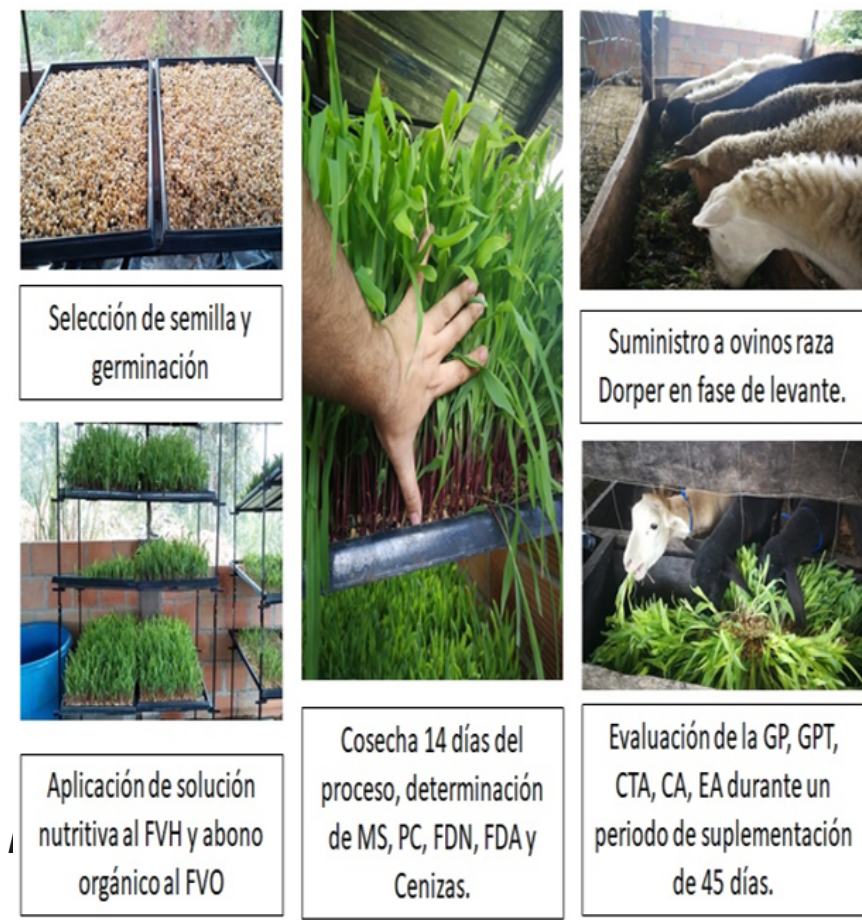

Evaluación de la GP, GPT,

CTA, CA, EA durante un

periodo de suplementación

de 45 días.
Composición nutricional del alimento base y de los suplementos FVHy

FVO a 14 días de proceso

\begin{tabular}{cccccc}
\hline Material & MS \% & PC \% & Cen \% & FDN \% & FDA \% \\
\hline DB & $17.53^{\mathrm{b}}$ & $11.57^{\mathrm{b}}$ & $12.24^{\circ}$ & $60.72^{\mathrm{b}}$ & $31.06^{\mathrm{b}}$ \\
FVH & $11.54^{\mathrm{a}}$ & $16.65^{\mathrm{a}}$ & $6.54^{\mathrm{b}}$ & $42.13^{\mathrm{a}}$ & $18.89^{\mathrm{a}}$ \\
FVO & $11.48^{\mathrm{a}}$ & $15.75^{\mathrm{a}}$ & $5.41^{\mathrm{a}}$ & $43.24^{\mathrm{a}}$ & $19.27^{\mathrm{a}}$ \\
\hline
\end{tabular}

Comportamiento productivo de ovinos en fase de levante suplementadoscon FVH y FVO.

\begin{tabular}{|c|c|c|c|c|c|}
\hline Variable & $\begin{array}{l}\text { T0 } \\
\text { (DB) }\end{array}$ & $\begin{array}{c}\mathrm{T} 1 \\
\text { (FVH) }\end{array}$ & $\begin{array}{c}\text { T2 } \\
\text { (FV0) }\end{array}$ & $\mathrm{EE}$ & $p$ \\
\hline Peso inicial & 13.05 & 13.06 & 13.01 & - & - \\
\hline Peso final & 15.40 & 16.72 & 16.62 & - & - \\
\hline Ganancia de peso total GPT $(\mathrm{kg})$ & $2.35^{3}$ & $3.66^{\mathrm{b}}$ & $3.61^{b}$ & 0.28 & 0.0142 \\
\hline $\begin{array}{c}\text { Ganancia diaria de peso } \\
\text { (GDP g/dia) }\end{array}$ & $52.22^{\mathrm{a}}$ & $81.39^{b}$ & $80.30^{b}$ & 6.21 & 0.0141 \\
\hline $\begin{array}{l}\text { Consumo Total de alimento } \\
\text { (CTA kg MS/dia) }\end{array}$ & $1.05^{\mathrm{a}}$ & $1.33^{\circ}$ & $1.27^{b}$ & 0.05 & 0.0012 \\
\hline Conversión alimenticia (CA) & $5.13^{3}$ & $3.23^{b}$ & $3.27^{b}$ & 0.37 & 0.0089 \\
\hline Eficiencia alimentaria (EA) & $0.050^{\mathrm{a}}$ & $0.063^{\mathrm{a}}$ & $0.061^{\mathrm{a}}$ & 0.02 & 0.1826 \\
\hline
\end{tabular}

\section{INTRODUCCIÓN}

El sector ganadero en el departamento del Putumayo es de gran importancia para la economía de la región. La población de ovinos en el año 2020 ascendió a 4306 animales (Instituto Colombiano Agropecuario [ICA], 2020), siendo una población relativamente baja en comparación con el inventario bovino. Se considera a la ovinocultura como una actividad productiva en crecimiento, generadora de ingresos $y$, desde el punto de vista social, puede tomarse como una iniciativa de reconversión productiva y de impulso a pequeños productores que no cuentan con áreas extensas de terreno para pastoreo.

Relacionado con lo anterior, la producción de forrajes en el trópico se ha convertido en una de las principales limitantes para la alimentación animal. Las pasturas tropicales, obtenidas en los suelos amazónicos, se caracterizan por presentar bajos rendimientos productivos y baja calidad nutricional. Por lo tanto, los ganaderos se ven en la necesidad de buscar nuevas alternativas que permitan mejorar la oferta alimenticia (Ramírez y Soto, 2017). Las variaciones climáticas, comúnmente presentadas en zonas tropicales, conducen a la baja calidad nutricional de la base forrajera utilizada en la producción pecuaria. Esta es la razón por la cual se utilizan dietas suplementarias (Espinoza et al., 2004). Sin embargo, la suplementación con alimentos comerciales crea una alta dependencia de insumos externos que limitan la rentabilidad del productor. Por lo anterior, la producción de forraje de manera no convencional se convierte en una alternativa viable para obtener alimento de alto valor nutricional en pequeños espacios y a bajo costo (Gamero, 2015).

Una alternativa para contrarrestar la baja oferta forrajera es la implementación de los cultivos sin suelo, en estos se germinan semillas de gramíneas y leguminosas de forma rápida y con buenos rendimientos en producción de biomasa verde. El maíz ha sido uno de los principales materiales utilizados para este proceso. Como resultado de este tipo de plantaciones están la producción de forraje verde hidropónico (FVH) y de forraje verde organopónico (FVO), que se realizan por medio de labores livianas que no demandan demasiado tiempo y fuerza de trabajo. Este sistema tiene como una de sus ventajas optimizar el uso de espacios reducidos y de suelos no aptos para producción de forrajes u otros cultivos (Guerrero y Narváez 2019). Se considera, además, como una 
actividad complementaria y no competitiva con la producción convencional de forrajes (Organización de las Naciones Unidas para la Agricultura y la Alimentación [FAO], 2001). Adicionalmente, se ha demostrado que este tipo de suplementación incrementa el consumo de alimento, la degradabilidad ruminal y digestibilidad total en el ganado ovino y caprino (Boccanera, 2017).

La producción de $\mathrm{FVH}$, específicamente, permite suministrar alimento de alto valor nutricional de manera constante (Paipa et al., 2020), se puede producir en lugares con condiciones climatológicas adversas o en sitios donde no se cuenta con oferta permanente de forraje verde (Martínez, 2019). Desde el punto de vista económico, Narváez et al. (2019) aseguran que la inversión inicial para el montaje y producción de FVH puede resultar elevado; sin embargo, los beneficios a corto, mediano y largo plazo son superiores en términos de rendimiento productivo. Ante dichas circunstancias, el objetivo de este estudio fue evaluar el efecto de la suplementación con FVH y FVO en ovinos en la fase de levante, en el piedemonte amazónico del Departamento del Putumayo.

\section{MATERIALES Y MÉTODOS}

\section{Localización}

La investigación se realizó en las instalaciones del Criadero Ovino Campanita S.A.S - Corderos de la Amazonía, ubicado en la Vereda Los Guaduales del Municipio de Mocoa, departamento del Putumayo, entre las coordenadas $01^{\circ} 05^{\prime} 16^{\prime \prime}$ latitud norte y $076^{\circ} 37^{\prime}$ 53" latitud sur, con una altitud de $670 \mathrm{msnm}$, temperatura promedio de $24^{\circ} \mathrm{C}$, humedad relativa del $85 \%$ y una precipitación de $4135 \mathrm{~mm}$ anuales con 150 a 200 días de Iluvia (Instituto de Hidrología, Meteorología y Estudios Ambientales [IDEAM], 2018) condiciones que corresponden a la zona vida de bosque muy húmedo tropical (Holdridge, 1978).

\section{Área experimental}

La producción de FVH y FVO se realizó en un invernadero de $72 \mathrm{~m}^{2}$ con cubierta en policarbonato de $3.5 \mathrm{~m}$ de altura y paredes laterales en ladrillo de $1.2 \mathrm{~m}$ de alto. Debido a las condiciones climáticas de la zona se cubrió con malla polisombra del $35 \%$, desde la base hasta la cubierta, con el objetivo de controlar plagas, roedores y aves que pudieran afectar los cultivos. En el interior se colocaron estructuras metálicas desarmables de $1,20 \mathrm{~m}$ de ancho y $1,2 \mathrm{~m}$ largo $\times 1,8$ $\mathrm{m}$ de alto, con capacidad para soportar 20 bandejas de polietileno (de $59 \mathrm{~cm}$ de largo $x$ $80 \mathrm{~cm}$ de ancho) de color negro, acanaladas y perforadas, en las cuales se realizó el proceso de germinación.

\section{Manejo del cultivo}

La producción de FVH se inició con la desinfección de las semillas (maíz amarillo, hibrido, FNC 115) con una solución de hipoclorito de sodio al 0,05 \% durante 2 minutos. Posteriormente se realizó el proceso de pregerminación, que consistió en dejar las semillas durante 24 horas en agua. Transcurrido este tiempo se realizó la siembra con una proporción de $2,2 \mathrm{~kg}$ de maíz $/ \mathrm{m}^{2}$. Las bandejas se cubrieron con un plástico negro durante 24 horas. Pasado este periodo se descubrieron y se proporcionó riego constante con la solución nutritiva planteada por Ramírez y Soto (2017) (Tabla 1) hasta el día 14 del proceso, en el que se realizó la cosecha.

Tabla 1. Composición de la solución nutritiva elementos mayores para 1000 litros.

\begin{tabular}{lccccc} 
& $\mathrm{N}$ & $\mathrm{P}_{2} \mathrm{O}_{5}$ & $\mathrm{~K}_{2} \mathrm{O}$ & $\mathrm{Ca}$ & $\mathrm{Mg}$ \\
$\mathrm{Ppm}$ & $\mathrm{ppm}$ & $\mathrm{ppm}$ & $\mathrm{ppm}$ \\
$\begin{array}{l}\text { Solución } \\
\text { Nutritiva }\end{array}$ & 208 & 65 & 242 & 212 & 40 \\
\hline
\end{tabular}

\begin{tabular}{|c|c|c|c|c|c|c|c|c|}
\hline & $\begin{array}{c}\text { S } \\
\text { ppm }\end{array}$ & $\begin{array}{c}\text { Fe } \\
\text { ppm }\end{array}$ & $\begin{array}{c}\text { Mn } \\
\text { ppm }\end{array}$ & $\begin{array}{c}\text { B } \\
\text { ppm }\end{array}$ & $\begin{array}{c}\text { Cu } \\
\text { ppm }\end{array}$ & $\begin{array}{l}\text { Zn } \\
\text { ppm }\end{array}$ & $\begin{array}{l}\text { Mo } \\
\text { ppm }\end{array}$ & $\begin{array}{c}\text { Cl } \\
\text { ppm }\end{array}$ \\
\hline $\begin{array}{l}\text { Solución } \\
\text { Nutritiva }\end{array}$ & 1.4 & 2.8 & 0.13 & 0.12 & 0.10 & 0.11 & 0.003 & 0.33 \\
\hline
\end{tabular}


Para la producción del FVO se cumplieron exactamente los pasos antes mencionados, con la salvedad de que (sobre la bandeja) se aplicó una capa de $0,3 \mathrm{~cm}$ de abono orgánico tipo lombricompost (Tabla 2), el cual sirvió como sustrato para el cultivo. Se realizaron riegos periódicos sin la adición nutrientes. Los forrajes se cosecharon a los 14 días cuando alcanzaron una altura promedio de $27 \mathrm{~cm}$.

Tabla 2. Composición del Lombricompost.

\begin{tabular}{ccccccccc} 
& $\mathrm{N}$ & $\mathrm{P}_{2} \mathrm{O}_{5}$ & $\mathrm{~K}_{2} \mathrm{O}$ & $\mathrm{CaO}$ & $\mathrm{MgO}$ & $\mathrm{S}$ & $\mathrm{B}$ & $\mathrm{Fe}$ \\
$\%$ & $\%$ & $\%$ & $\%$ & $\%$ & $\%$ & $\%$ & $\%$ \\
$\begin{array}{c}\text { Solución } \\
\text { Nutritiva }\end{array}$ & 2.49 & 0.53 & 4.52 & 2.08 & 1.68 & 0.21 & 0.008 & 0.75 \\
\hline
\end{tabular}

Fuente: Autores

\section{Producción de biomasa}

Posterior a los 14 días de germinación, tanto para FVH y FVO, se determinó la producción de biomasa total y se midió la altura, siguiendo la metodología descrita por Vargas-Rodríguez (2008).

\section{Calidad nutricional}

Durante la fase experimental se recolectaron muestras de $\mathrm{FVH}$ y FVO directamente de las bandejas de germinación, antes del suministro a los animales. Las muestras se trasladaron al Laboratorio de aguas, suelos y biotecnología del Centro Agroforestal y Acuícola Arapaima del Sena, Regional Putumayo, en el que se secaron en una estufa de ventilación forzada a una temperatura de $65^{\circ} \mathrm{C}$ durante 72 horas. Las muestras fueron molidas por medio de un molino estacionario con una criba de $1 \mathrm{~mm}$ $y$, posteriormente, enviadas al laboratorio de nutrición animal del Centro de Investigación Tibaitata de Agrosavia. Allí se determinó el porcentaje de Materia Seca (MS), Proteína Cruda (PC), Cenizas, Fibra detergente neutro (FDN) y Fibra detergente acido (FDA) en las muestras, mediante la técnica de espectroscopía de reflectancia en infrarrojo cercano (NIRS) (Ariza-Nieto et al., 2018).

\section{Animales experimentales}

Se seleccionaron 12 corderos machos enteros de la raza Dorper en fase de levante (90 días de edad \pm 5 días), con un peso promedio inicial de $14,62 \mathrm{~kg}$, los cuales se distribuyeron para tres tratamientos. Previo al inicio del experimento, los animales se desparasitaron con un producto comercial a base de Fenbendazol y se alojaron en corrales individuales provistos de comederos y bebederos con agua ad libitum, en los que tuvieron un periodo de adaptación de 12 días. La fase experimental tuvo una duración de 45 días, en los que el nivel de suplementación fue del $6.9 \%$ del PV con las dietas propuestas.

\section{Tratamientos y diseño experimental}

Los tratamientos evaluados fueron: T0 (Dieta base): $100 \%$ Forraje de Pennisetum purpureum; T1: $70 \%$ Pennisetum purpureum + $30 \%$ de FVH; y T2: $70 \%$ Pennisetum purpureum + $30 \%$ FVO. Se utilizó un diseño completamente al azar, con cuatro repeticiones por tratamiento, en los que la unidad experimental consistió en un cordero, dando un total de 12 animales.

\section{Variables evaluadas}

Ganancia de peso total (GPT) y Ganancia Diaria de Peso (GDP). LoS ovinos se pesaron individualmente al inicio y semanalmente hasta el final del ensayo, con una báscula electrónica de brazo marca Fuller Machinery (BASC-SHR5), y, mediante estas mediciones, se estimó la GPT a través de la siguiente ecuación: GPT = Peso final - Peso inicial; mientras que la ganancia diaria de peso (GDP) se calculó mediante la ecuación GDPD = (Peso final Peso inicial) / Número de días experimentales.

Consumo total de Alimento (CTA). El consumo de alimento se determinó mediante la diferencia entre el forraje ofrecido y el residual aplicando la siguiente ecuación: CTA = (alimento suministrado - alimento rechazado) (Saguilán et al., 2020).

Conversión alimenticia (CA) y eficiencia alimenticia (EA): La conversión 
alimenticia (CA) se estimó mediante la relación entre el consumo de alimento $(\mathrm{Kg})$ y la ganancia diaria de peso $(\mathrm{kg})$ (Sanchez et al. 2009), mientras que la eficiencia alimenticia (EA) se estableció mediante la GDP y el consumo de materia seca $\mathrm{Kg} / \mathrm{MS}$ (Pérez et. al., 2011).

Análisis estadístico. Una vez se verificó que los datos cumplieron con los supuestos de normalidad y homogeneidad, tras aplicar las pruebas de Shapiro y Bartlett, se realizó un análisis de varianza (ANOVA), ajustado a un Diseño Completamente al Azar (DCA) por medio del paquete estadístico Minitab 16 (Minitab, s.f.). Las fuentes de variación fueron: modelo, tratamientos y error. Para las comparaciones entre los tratamientos se utilizó la prueba de Tukey, con una significancia de $5 \%$. El modelo matemático que describió el diseño fue:

$$
Y i j=\mu+T j+e i j
$$

En el que Yij es el efecto del tratamiento; $\boldsymbol{\mu}$ es la media general; $\mathbf{T} \mathbf{j}$ es el efecto del $\mathbf{j}$ esimo tratamiento; e es el error aleatorio de la ij-ésima repetición que recibió el j-ésimo tratamiento.

\section{RESULTADOS Y DISCUSIÓN}

\section{Producción de biomasa}

El mayor rendimiento en producción de biomasa se obtuvo en el $\mathrm{FVH}$, con $6.36 \mathrm{~kg}$ de forraje por $\mathrm{kg}$ de semilla; por su parte, el FVO alcanzó 5.24 kg, encontrándose diferencias $(p<0.05)$ en los dos modelos de obtención de forraje verde evaluados (Tabla 3 ). De acuerdo con Vargas-Rodríguez (2008), el rendimiento óptimo en la producción de forrajes, usando sistemas hidropónicos, se da cuando la relación entre la biomasa obtenida y la semilla germinada es de 5:1, para el FVH la relación encontrada fue de 6.36:1 y para el
FVO de 5.24:1. Esta relación en la producción de forraje verde puede variar dependiendo de la calidad de semilla y de la solución nutritiva empleada en el proceso, indicando que el aporte de la solución de macro y micronutrientes utilizada en la producción de FVH fue más completa que el aporte de nutrientes del cultivo organopónico de maíz proveniente del abono orgánico.

La producción de biomasa en el cultivo hidropónico fue similar a la reportada por Osorno y González (2012) y Tarrillo (2007), quienes aseguran que se puede obtener de 6 a $8 \mathrm{~kg}$ de masa forrajera totalmente consumible a partir de $1 \mathrm{~kg}$ de semilla. Elizondo (2005) relaciona producciones de hasta $9 \mathrm{~kg}$ de biomasa, sin embargo, en la presente investigación no se logró obtener tales rendimientos. Por otra parte, Espinosa (2019) reporta que a los 12 días de cosecha el maíz hibrido alcanza un rendimiento de $4.4 \mathrm{~kg}$ de forraje. Se debe considerar que la semilla utilizada en este estudio fue un maíz hibrido FNC 115, en el que el FVO no se recibió solución nutritiva, evidenciando un alto rendimiento de biomasa $(5.24 \mathrm{~kg}$ ) a partir de abono orgánico (tipo lombricomposta) como sustrato.

El medio de cultivo no influyó en la altura de las plantas de maíz ( $p>0.05)$, registrando valores de $27.80 \mathrm{~cm}$ y 27.48 $\mathrm{cm}$ respectivamente para FVH y FVO (Tabla. 3). La altura de las plantas producidas en sistemas hidropónicos u organopónicos se relaciona directamente con el rendimiento en producción de biomasa vegetal y se considera como un criterio de cosecha cuando esta alcanza los 25 cm (Bombana y Gai, 2019). En el presente estudio, a los 14 días de germinación la altura fue superior al valor de referencia antes mencionado, posiblemente por las condiciones donde se realizaron los montajes y por el tipo de semilla empleada.

Tabla 3. Producción de biomasa de maíz a los 14 días de establecido.

\begin{tabular}{ccccc}
\hline Variable & FVH & FVo & Estadístico T & P \\
Biomasa $\left(\mathrm{kg}^{*}\right)$ & $6.36 \mathrm{a}$ & $5.24 \mathrm{~b}$ & 3.88 & 0.0047 \\
Altura $(\mathrm{cm})$ & $27.80 \mathrm{a}$ & $27.48 \mathrm{a}$ & 0.70 & 0.4992
\end{tabular}


Ramírez y Soto (2017) reportan una altura promedio de $28.13 \mathrm{~cm}$ en FVH de maíz, con dos niveles de fertilización mineral en 11 días de proceso. Por otra parte, Morales et al. (2012) encontraron a los 8 días de cosecha una altura de $19.5 \mathrm{~cm}$ y Vargas-Rodríguez (2008) asegura que a los 12 días de cosecha la altura fue de $22 \mathrm{~cm}$. De igual manera, Chavarria-Torrez y Castillo-Castro (2018) reportan una altura promedio de $25 \mathrm{~cm}$ a los 14 días del proceso. La importancia de considerar la altura radica en las necesidades del productor, ya que a mayor altura habrá mayor biomasa verde disponible para cubrir los requerimientos de los animales.

\section{Composición nutricional del alimento base y de los suplementos FVH y FVO.}

En la Tabla 4 se presentan los resultados del análisis bromatológico de los tratamientos evaluados. Se encontraron diferencias ( $p$ $<0.05$ ) para el contenido de materia seca (MS) entre el FVH y FVO con la dieta base. La MS del material vegetal obtenido mediante sistemas hidropónicos tiende a aumentar con los días de germinación; sin embargo, la gramínea Pennisetum purpureum (DB) presento el mayor contenido de MS con
$17.53 \%$, debido posiblemente a la edad de corte que presentaba el cultivo (45 días), seguida del FVH con $11.54 \%$ y del FVO con $11.48 \%$. La MS de los forrajes tiene importantes implicaciones desde el punto de vista nutricional y alimenticio, ya que en la medida que aumenta el contenido de MS, aumenta la densidad de los nutrientes (Naranjo y Cuartas, 2011).

Con respecto al porcentaje de $\mathrm{PC}$, se encontraron diferencias $(p<0,05)$ entre la dieta base y los suplementos evaluados. El mayor contenido de PC se encontró en el FVH con $16.65 \%$, relacionándose posiblemente con la fertilización a base de la solución de micro y macronutrientes, mientras que el FVO presentó una PC de $15.75 \%$, evidenciando que existe una relación proporcional entre el tipo de fertilización y el contenido de proteína del forraje. Los valores encontrados en el presente estudio fueron superiores a los reportados por Salas-Pérez et al. (2010) quienes, al utilizar solución nutritiva para la producción de $\mathrm{FVH}$ de maíz, obtuvieron $13.25 \%$ de PC, mientras que, al aplicar té de composta, los tenores proteicos reportados disminuyeron a un $13 \%$.

Tabla 4. Composición nutricional del alimento base y de los suplementos FVH y FVO a los 14 días de proceso

$\begin{array}{cccccc}\text { Material } & \text { MS \% } & \text { PC \% } & \text { Cen \% } & \text { FDN \% } & \text { FDA \% } \\ \text { DB } & 17.53 \mathrm{a} & 11.57 \mathrm{~b} & 12.24 \mathrm{a} & 60.72 \mathrm{a} & 31.06 \mathrm{a} \\ \text { FVH } & 11.54 \mathrm{~b} & 16.65 \mathrm{a} & 6.54 \mathrm{~b} & 42.13 \mathrm{~b} & 18.89 \mathrm{~b} \\ \text { FVO } & 11.48 \mathrm{~b} & 15.75 \mathrm{a} & 5.41 \mathrm{c} & 43.24 \mathrm{~b} & 19.27 \mathrm{~b}\end{array}$

Letras distintas indican diferencias significativas $(p<0,05)$

MS: Materia seca, PC: Proteína cruda, Cen: Cenizas, FDN: Fibra detergente neutro, FDA: Fibra detergente acido DB: Forraje de Pennisetum purpureum

Fuente: autores

En el presente estudio se obtuvieron valores de proteína superiores para el FVH y FVO en comparación con la dieta base, indicando que es recomendable la implementación de estos suplementos en sistemas de producción de pequeños rumiantes. Trevizan y Challapa (2020), compararon el rendimiento productivo del FVH del maíz criollo vs maíz comercial, encontrando un contenido de PC máximo de $15.38 \%$. Por otra parte, Chavarria-Torrez y Castillo-Castro (2018) reportan contenidos de PC de $16 \%$, y López-Aguilar et al. (2009) entre $15,2 \%$ y $15,8 \%$ para FVH de maíz cosechado a los 14 días. Sin embargo, es importante considerar que el contenido de PC de estos forrajes tiende a disminuir, a medida que avanza la edad de maduración del cultivo, Müller et al. (2005) encontraron contenidos de PC en FVH de maíz a los 12 días de 17.4 $\%$, y de $13.4 \%$ a los 14 días, demostrando una disminución significativa.

La aplicación de solución nutritiva afecto el contenido de cenizas del FVH en comparación con el FVO, el análisis estadístico demostró diferencias $(p<0.05)$ en la concentración 
mineral de los cultivos. La cantidad de cenizas fue de $6.54 \%$ y $5.41 \%$ para FVH y FVO respectivamente, mientras que la dieta base presento un porcentaje de $12.24 \%$. Los resultados aquí encontrados coinciden con Maldonado et al. (2013), quienes aseguran que la cantidad de cenizas que contiene el forraje se incrementan con el suministro de nitrógeno. Esto demuestra la influencia de la solución macro y micromineral empleada en la producción del FVH. Sin embargo, los resultados obtenidos para el FVO, el cual recibió una fertilización orgánica, demostraron que el rendimiento productivo en biomasa y altura son similares a los obtenidos con una fertilización que contenga soluciones nutritivas. Ramírez y Soto (2017) afirman que no es necesario el uso de soluciones nutritivas en la producción de FHV para cosechas de 11 días, evidenciando el uso de fertilizantes orgánicos como alternativa útil de producción de cultivos hidropónicos y organopónicos.

Con relación a las fracciones de fibra, el tratamiento que mayor porcentaje presentó fue el T0 (dieta base) con $60.72 \%$ FDN y $31.06 \%$ FDA. El análisis de varianza demostró diferencias estadísticas $(p<0.05)$ entre los tratamientos T1 (FVH) y T2 (FVO) con la dieta base analizada. Entre los suplementos (FVH y FVO) no se encontraron diferencias para los contenidos de FDN y FDA. Los resultados obtenidos confirman que la cantidad de fibra en las plantas jóvenes es relativamente baja si se compara con plantas maduras, ya que la estructura de la fibra tiende a cambiar a medida que la edad de la planta avanza, debido a que aumenta el contenido de lignina y por lo tanto su digestibilidad tiende a disminuir. Posiblemente, esto está relacionado con el bajo rendimiento en las variables de productividad animal presentadas por la dieta base, en comparación con la inclusión de 30 \% de FVH y FVO de maíz para la alimentación de ovinos en fase de levante.

En síntesis, de acuerdo con lo encontrado en el presente estudio, la suplementación con FVH y FVO de maíz, por ser alimentos con elevado aporte de nutrientes, los convierten en una alternativa viable para implementarse en los sistemas de producción, permitiendo, de esta forma, corregir las deficiencias nutricionales de los forrajes empleados en pastoreo.

\section{Ganancia de peso total (GPT) y ganancia diaria de peso (GDP).}

La suplementación con FVH y FVO de maíz influyó en el desempeño productivo de los animales $(p<0.05)$. En este sentido, los ovinos que recibieron FVH y FVO registraron una GPT de 3.66 y $3.61 \mathrm{~kg}$ respectivamente, difiriendo de las ganancias registradas en los animales del T0 $(2.35 \mathrm{~kg})$. Así mismo, se observó que las ganancias diarias de peso fueron mayores $(p<0.05)$ en los animales que recibieron $\mathrm{FVH}$ y $\mathrm{FVO}$, los cuales presentaron valores medios de 81.39 y $80.30 \mathrm{~g} /$ día, respectivamente. En cuanto al consumo total de alimento (CTA), conversión alimenticia (CA) y eficiencia alimenticia (EA), el análisis también detectó diferencias ( $p<$ $0,05)$, siendo los animales suplementados con FVH y FVO los que expusieron los mayores consumos, y mejores conversión y eficiencia alimenticia (Tabla 5).

Tabla 5. Comportamiento productivo de ovinos en fase de levante suplementados con FVH y FVO.

\begin{tabular}{|c|c|c|c|c|c|}
\hline Variable & $\begin{array}{l}\mathrm{TO} \\
(\mathrm{DB})\end{array}$ & $\begin{array}{c}\text { T1 } \\
(\mathrm{FVH})\end{array}$ & $\begin{array}{l}\text { T2 } \\
(\mathrm{FVO})\end{array}$ & EE & P \\
\hline Peso inicial & 13.05 & 13.06 & 13.01 & - & - \\
\hline Peso final & 15.40 & 16.72 & 16.62 & - & - \\
\hline $\begin{array}{c}\text { Ganancia de peso total GPT }(\mathrm{kg}) \\
\text { Ganancia diaria de peso }\end{array}$ & $2.35 \mathrm{~b}$ & $3.66 \mathrm{a}$ & $3.61 \mathrm{a}$ & 0.28 & 0.0142 \\
\hline $\begin{array}{l}\text { (GDP g/día) } \\
\text { Consumo Total de alimento }\end{array}$ & $52.22 b$ & $81.39 \mathrm{a}$ & $80.30 \mathrm{a}$ & 6.21 & 0.0141 \\
\hline (CTA kg MS/día) & $1.05 \mathrm{~b}$ & $1.33 \mathrm{a}$ & $1.27 \mathrm{a}$ & 0.05 & 0.0012 \\
\hline Conversión alimenticia (CA) & $5.13 \mathrm{a}$ & $3.23 \mathrm{~b}$ & $3.27 \mathrm{~b}$ & 0.37 & 0.0089 \\
\hline Eficiencia alimentaria (EA) & $0.050 \mathrm{a}$ & $0.063 \mathrm{a}$ & $0.061 \mathrm{a}$ & 0.02 & 0.1826 \\
\hline
\end{tabular}

Letras distintas indican diferencias significativas $(p<0,05)$

EE: Error estándar

Fuente: Autores 
El suministro de FVH de maíz aporta proteínas, minerales y vitaminas altamente asimilables porque estos nutrientes no se encuentran tan solubles en el grano seco. Las vitaminas y minerales juegan un papel importante en la fertilidad y productividad, sumado al aporte proteico que se refleja en la ganancia de peso total de los animales. En los sistemas de alimentación de ovinos en los que se incluyó el $30 \%$ de $\mathrm{FVH}$ se reportan ganancias de peso de hasta $4.82 \mathrm{~kg}$ (Castellón y Torrez, 2018), superiores a las encontradas en esta investigación. Por otra parte, Sánchez et al. (2013) informan que los corderos pueden llegar a consumir hasta el $3.7 \%$ de su peso vivo en materia seca de FVH. Sin embargo, Arano (1998) menciona que la dieta debe complementarse con una fuente de fibra para evitar problemas de timpanismo.

El contenido de PC no difiere en gran medida en los dos suplementos probados (16.65 \% FVH y $15.75 \%$ FVO). Sin embargo, la composición nutricional del FVH tiende a ser mejor, lo que podría sugerir una mayor producción de ácidos grasos volátiles a nivel ruminal, atribuyendo la diferencia en la GDP, entre los tratamientos evaluados, a la composición nutricional de los suplementos. Acosta-Lozano et al. (2018) reportan ganancias diarias de peso superiores, en caprinos en fase de levante - ceba, tras incluir biomasa hidropónica de maíz $(84,54$ $\mathrm{g} /$ día), en comparación con los caprinos alimentados con un suplemento a base de maíz y concentrado comercial (81,71 g/ día). Los resultados anteriores indican que los suplementos compuestos por FVH y FVO, por ser alimentos de alta degradabilidad, pueden contribuir a mejorar la actividad de las bacterias celulolíticas $y$, de esta manera, mejorar la relación energía-proteína disponible en el rumen, favoreciendo el crecimiento microbiano y los productos de la fermentación ruminal (Naik et al., 2014).

\section{Consumo total de alimento (CTA)}

Se encontraron diferencias estadísticas para la variable Consumo Total de Alimento (CTA) $(p<0.05)$. El mayor consumo $(1.33 \mathrm{~kg}$ $\mathrm{MS} /$ día) se obtuvo en el tratamiento T1 (FVH) y fue similar $(p>0.05)$ al obtenido en el T2 (1.27 kg MS/día), pero diferente y superior a $(p<0.05)$ a los valores encontrados en el tratamiento T0 (1.05 kg MS/día). Estas diferencias en el CTA se pueden atribuir a la palatabilidad del $\mathrm{FVH}$, al ser un producto del desdoblamiento del almidón en azucares simples durante el proceso de germinación (Azócar et al., 2007).

El consumo de materia seca se puede ver afectado por las variables de la composición nutricional de la dieta, ya que, cuando el contenido de fibra es elevado, el alimento se vuelve poco apetecible para el animal. Las fracciones de FDN presentes en el FVH (T1) fueron de $42.13 \%$ y $43.24 \%$ para el FVO (T2); mientras que la dieta base (T0) presentó una FDN de $60.72 \%$, corroborando la importancia del contenido de fibra en el consumo total de alimento. Herrera et al. (2007) reportaron un consumo de MS promedio de $0.489 \mathrm{~kg} /$ día al evaluar el efecto de la suplementación con forraje hidropónico de maíz en ovinos mestizos en crecimiento, los cuales fueron valores inferiores a los registrados en esta investigación. Por otra parte, Morales (2017) encontró que al incluir 40 y $50 \%$ de $\mathrm{FVH}$ en la dieta de ovinos de pelo, el consumo de MS fue de 1.32 y $1.50 \mathrm{~kg} /$ día respectivamente, indicando que la suplementación con el 30 $\%$ FVH y/o FVO de maíz permite obtener un CTA superior al que presentan los animales en pastoreo sin ningún tipo de alimentación complementaria.

\section{Conversión alimenticia (CA) y eficiencia alimenticia (EA)}

Con relación a la conversión alimenticia (CA), se encontraron diferencias estadísticas entre los tratamientos T1 y T2 con valores de 3.23 y 3.27 , respecto al T0 con un valor de 5.13; la mejor CA se presentó en el T1 $(\mathrm{FVH})$, lo que sugiere que, por cada $\mathrm{kg}$ de peso que ganaron los ovinos, su consumo fue de $3.23 \mathrm{~kg}$ de alimento. Los resultados encontrados en esta investigación coinciden con los datos obtenidos por Castellón y Torrez (2018), quienes reportan una CA de 3.55 al incluir $30 \%$ de FVH de maíz en la dieta de ovinos; mientras que en un sistema de alimentación basado en el $100 \%$ de pastoreo de gramíneas, la conversión alimenticia fue de 8.99. Por otra parte, Acosta (2016) reportó una CA de 7.12 en animales alimentados con concentrado comercial y maíz. Hay que anotar que valores bajos de CA indican que el rendimiento productivo será superior, ya que 
se requiere una menor cantidad de alimento para ganar $1 \mathrm{~kg}$ de peso (Águila, 2020). De esta manera, se recomienda incluir, en un 30 $\%$ del total de la dieta de los ovinos, planes de suplementación basados en el suministro de FVH y FVO de maíz, otros cereales y demás materiales disponibles en la zona donde se desarrolle la actividad productiva.

Teniendo en cuenta las anteriores consideraciones, se puede afirmar que la inclusión de FVH como suplemento nutricional, en las dietas convencionales basadas en el consumo de forrajes principalmente gramíneas, es una de las alternativas productivas más eficientes en la actualidad (Salas-Pérez et al. 2010); ya que, al igual que el FVO, se produce en espacios reducidos, la dependencia de insumos externos es mínima y las variaciones climáticas no afectan su producción, manteniendo una oferta permanente de alimento de alto valor nutricional.
La eficiencia alimentaria (EA) no presentó diferencias estadísticas entre los tratamientos evaluados, encontrándose para el T1 (FVH) un valor de $0.063 ; 0.061$ para el T2 (FVO); y para la dieta base (T0) un valor de EA de 0.050; indicando que la ganancia de peso no fue lo suficientemente significativa con relación al consumo de alimento. Unos resultados similares reportaron Ruiz-Sesma et al. (2006) al evaluar el comportamiento productivo de ovinos de pelo, con la inclusión de diferentes niveles de heno de Hibiscus rosa-sinensis, obteniendo una EA promedio de 0.079. Por otra parte, Pérez et al. (2011), al evaluar un sistema de alimentación intensiva en condiciones de estabulación con ovinos de pelo, reportan una eficiencia alimenticia de 0.193 . Mostrando, de esta manera, que es necesario ofrecer a los ovinos una dieta rica en carbohidratos solubles, que estén disponibles para la microbiota ruminal, y de esta manera cubrir de manera eficiente los requerimientos energéticos de mantenimiento y crecimiento en los animales.

\section{CONCLUSIONS}

El tipo de fertilización no tuvo influencia significativa en la altura de los forrajes, teniendo en cuenta que uno de los criterios más importantes para la cosecha es la altura de la planta (cuando esta alcanza los $25 \mathrm{~cm}$ ).

Los dos tipos de producción de forraje verde (hidropónico y organopónico) presentaron valores superiores: $27.80 \mathrm{~cm}$ para $\mathrm{FVH}$ y $27.48 \mathrm{~cm}$ para FVO, indicando que el FVO es una alternativa viable para implementarse como suplemento nutricional para ovinos, ya que fomenta el uso de abonos orgánicos y así propende por sistemas sostenibles de producción animal.

El análisis bromatológico demostró que MS, PC, FDN y FDA no presentan diferencias en los dos sistemas de producción de forraje, infiriendo que la solución nutritiva empleada en la producción de FVH no afecto su composición nutricional. Sin embargo, el contenido de cenizas sí se afectó por la solución nutritiva, corroborando que los minerales del forraje se incrementan cuando la fertilización nitrogenada es alta.

La inclusión del $30 \%$ de FVH o FVO de maíz en la dieta de ovinos en fase de levante, basada en el pastoreo de gramíneas tropicales, afectó de manera positiva el comportamiento productivo, mejorando la ganancia de peso diaria y total, sin afectar el consumo total de alimento, la conversión y eficiencia alimenticia. Por consiguiente, la producción de FVH y FVO se presenta como una alternativa factible de suplementación de los ovinos en condiciones tropicales. Sin embargo, se sugiere realizar más estudios que impliquen el uso de otras semillas y sustratos que permitan mejorar el rendimiento productivo del cultivo $y$, por ende, la respuesta animal. 


\section{CONTRIBUCIÓN DE LA AUTORÍA}

Juan Pablo Narváez Herrera: Investigación, metodología, análisis de datos, conceptualización, escritura, borrador original, revisión y edición. Elizabeth Marcela Guerrero Guerrero: Investigación, conceptualización, análisis de datos, escritura, borrador original.

\section{AGRADECIMIENTOS}

Los autores agradecen al Servicio Nacional de Aprendizaje [SENA], Centro Agroforestal y Acuícola Arapaima (Regional Putumayo), al Sistema de Investigación Desarrollo Tecnológico e Innovación [SENNOVA] y a la empresa criadero Ovino Campanita SAS - Corderos de la Amazonia, por facilitar sus instalaciones para realizar el trabajo de campo de esta investigación.

\section{LITERATURA CITADA}

Acosta, N. V. (2016). Evaluación de la biomasa hidropónica de maíz como alimento para caprinos criollos en crecimientoceba [Tesis Doctoral, Universidad Central "Marta Abreu" de Las Villas.]. DSpace@ UCLV. https://dspace.uclv.edu.cu/bitstream/ handle/123456789/7320/Nestor\%20Acosta_ Tesis $\% 20 \mathrm{PhD}$. pdf ? sequence $=1$ \&isAllowed $=\mathrm{y}$

Acosta-Lozano, N., Lima-Orozco, R., González-Asencio, M. F., Quinteros-Freire, C., Masaquiza-Moposita, D., Ayala, L., VargasBurgos, J., Ortiz-Nacaza, P. y Andrade-Yucailla, V. (2018). Comportamiento biproductivo de caprinos criollos en crecimiento-ceba alimentados con biomasa hidropónica de maíz en el Litoral ecuatoriano. Redvet, 19(7), 1-13. www.veterinaria.org/revistas/redvet/ n070718.html

Águila, R. (2020). La incomprendida conversión alimenticia. Porcicultura.com. https://www.porcicultura.com/destacado/ La-incomprendida-conversi\%C3\%B3nalimenticia

Arano, R. (1998). Forraje verde hidropónico y otras técnicas de cultivos sin tierra: una guía práctica para los interesados en la hidroponía moderna. Universidad de Buenos Aires.
Ariza-Nieto, C., Mayorga, O. L., Mojica, B., Parra, D. \& Afanador-Tellez, G. (2018). Use of LOCAL algorithm with near infrared spectroscopy in forage resources for grazing systems in Colombia. Journal of Near Infrared Spectroscopy, 26(1), 44-52. https:// www. osapublishing.org/jnirs/abstract. cfm?URI=jnirs-26-1-44

Azócar, P., Manterola, H., Mira, J., Rojo, H. y Bravo, S. (2007). Respuesta productiva de cabras criollas en lactancia, alimentadas con forraje hidropónico. Avances en producción animal, 32(1-2), 83-94. http://bibliotecadigital.ciren.cl/bitstream/ handle/123456789/31678/Respuesta\%20 productiva. pdf? sequence $=1$ \&isAllowed $=y$

Boccanera, M. (2017). Utilización de Forraje Verde Hidropónico en dietas para caprinos [Tesis de pregrado, Universidad Nacional de La Plata]. Sedici. http://sedici.unlp.edu.ar/bitstream/ handle/10915/61201/Documento_completo. pdf?sequence $=1$ \&isAllowed $=y$

Bombana, W. A., y Gai, V. F. (2019). Cultivo de milho hidropônico com diferentes adubações. Revista Cultivando o Saber, 12(4), 49-57. https://www.fag.edu.br/upload/ revista/cultivando_o_saber/5dfbdca228c37. pdf

Castellón, M. y Tórrez, F. (2018). Inclusión de forraje verde hidropónico en la alimentación de ovinos en desarrollo y su efecto en el comportamiento productivo, Finca Santa Rosa, Managua, 2017 [Tesis de pregrado, Universidad Nacional Agraria. Archivo digital. https://1library.co/document/ y863rrwq-inclusion-forraje-hidroponicoalimentacion-desarrollo-comportamientoproductivo-managua.html

Chavarria-Torrez, A. y Castillo-Castro, S. (2018). El forraje verde hidropónico (FVH), de maíz como alternativa alimenticia y nutricional para todos los animales de la granja. Revista Iberoamericana de Bioeconomía y Cambio Climático, 4(8), 1032-1039. https://doi. org/10.5377/ribcc.v4i8.6716

Elizondo, J. (2005). Forraje verde hidropónico: Una alternativa para la 
alimentación animal. ECAG-informa, (32), 36-39

Espinosa, W. (2019). Evaluación de densidades de siembra en maíz, arroz y frijol vigna en la producción de forraje verde hidropónico. Investigaciones Agropecuarias, 1(2), 15-27. https://revistas.up.ac.pa/index. php/investigaciones_agropecuarias/article/ view/493/403

Espinoza, F., Argenti, P., Urdaneta, G., Araque, C., Fuentes, A., Palma, J. y Bello, C. (2004). Uso del forraje de maíz (Zea mays) hidropónico en la alimentación de toretes mestizos. Zootecnia tropical, 22(4), 303-315. http://ve.scielo.org/scielo.php?script $=$ sci_ arttext\&pid =S0798-72692004000400001

Organización de las Naciones Unidas para la Agricultura y la Alimentación. (2001). Manual técnico: forraje verde hidropónico. Oficina Regional de la FAO para América latina y el Caribe.

Gamero, C. J. (2015). Evaluación de gramíneas y leguminosas de trópico alto en cultivo hidropónico como alternativa de producción orgánica para la nutrición animal [Tesis de pregrado, Universidad de la Salle]. Ciencia Unisalle. https://ciencia.lasalle.edu. co/zootecnia/215

Martínez, F. (2019). Forraje Verde Hidropónico ( $F . \quad$ V. H) para alimentación de animales. https://infopastosyforrajes. com/suplementacion/forraje-verdehidroponico/\#Descargar_el_Documento

Guerrero, E. y Narváez. J. (2019). Efecto de la suplementación con forraje verde hidropónico y germinado orgánicos en la alimentación animal. Entelequia, 1(3), 6.

Herrera, A. M., Depablos, L. A., López, R., Benezra, M. A. y Ríos, L. (2007). Degradabilidad y digestibilidad de la materia seca del forraje Hidropónico de Maíz (Zea Mays). Respuesta animal en términos de consumo y ganancia de peso. Revista científica, 17(4), 372-379. https://www. redalyc.org/articulo.oa?id=95917409
Holdridge, L. R. (1996). Ecología basada en las zonas de vida. - Instituto Interamericano de Ciencias Agrícolas. https://repositorio. iica.int/handle/11324/7936

Instituto Colombiano Agropecuario (2020). Censo Pecuario año 2020. https:// www.ica.gov.co/areas/pecuaria/servicios/ epidemiologia-veterinaria/censos-2016/ censo-2018

Instituto de Hidrología, Meteorología y Estudios Ambientales (2018). Subdirección de meteorología. estación seleccionada se ubica en las siguientes Coordenadas Geográficas: 04 $11^{\prime}$ latitud Sur, 6956' longitud Oeste, elevación: 84 m.

López-Aguilar, R., Murillo-Amador, B. y Rodríguez-Quezada, G. (2009). El forraje verde hidropónico $(\mathrm{FVH})$ : Una alternativa de producción de alimento para el ganado en zonas áridas. Interciencia, 34(2), 121-126. http://ve.scielo.org/scielo.php?script $=$ sci_ arttext\&pid=S0378-18442009000200009

Pérez, E. J., García, M. C., Albares, S., Sosa, R. y Velasco, H. L. (2011). Parámetros productivos de ovinos de pelo en un sistema de alimentación intensiva en la región central de Chiapas. Quehacer Científico en Chiapas, 1(12), 7-13. https:// www.dgip.unach.mx/images/pdf-REVISTAQUEHACERCIENTIFICO/QUEHACERCIENTIFICO-2011-jul-dic/Parametros_ productivos_de_ovinos_de_pelo.pdf

Maldonado, R., Álvarez, M., Acevedo, D. C. y Ríos, E. (2013). Nutrición mineral de forraje verde hidropónico. Revista Chapingo Serie horticultura, 19(2), 211-223. https:// doi.org/10.5154/r.rchsh.2011.10.053

Minitab. (s. f.). Minitab statistical software. www.minitab.com/en-us/products/minitab/

Morales, J. A. (2017). Evaluación del consumo de forraje verde hidropónico de maíz (Zea mays), en ovinos de pelo en desarrollo estabulados en el municipio de Chimaltenango, Chimaltenango [Tesis de 
pregrado, Universidad de San Carlos de Guatemala]. Repositorio institucional USAC. http://www.repositorio.usac.edu.gt/12415/

Morales, H. J., Gómez-Danés, A. A., Juárez, P., Loya, L. y de Coss, A. (2012). Forraje verde hidropónico de maíz amarillo (Zea maíz L.) con diferente concentración de solución nutritiva. Abanico veterinario, 2(3), 20-28.

Müller, L., Manfron, P. A., Santos, O. S., Medeiros, S. L. P., Haut, V., Neto, D. D., Fagan, E. V. y Bandeira, A. H. (2005). Produção e composição bromatologica da forragem hidropônica de milho, Zea mays L., com diferentes densidades de semeadura e datas de colheita. Zootecnia Tropical, 23(2), 105-119. http://ve.scielo.org/scielo.php?script $=$ sci_ arttext\&pid=S0798-72692005000200002

Naik, P. K., Dhuri, R. B., Karunakaran, M., Swain, B. K. \& Singh, N. P. (2014). Effect of feeding hydroponics maize fodder on digestibility of nutrients and milk production in lactating cows. Indian Journal of Animal Sciences, 84(8), 880-883. https://www. researchgate.net/publication/288748815 Effect_of_feeding_hydroponics_maize_ fodder_on_digestibility_of_nutrients_and_ milk_production_in_lactating_cows

Naranjo, J. F. y Cuartas, C. A. (2011). Caracterización nutricional y de la cinética de degradación ruminal de algunos de los recursos forrajeros con potencial para la suplementación de rumiantes en el trópico alto de Colombia. Revista CES Medicina Veterinaria y Zootecnia, 6(1), 9-19. https:// www.redalyc.org/pdf/3214/321428105002. pdf

Narváez, J., Gómez, G., Mena, C., Daza, L. y Bacca, S. (2019). Costos de producción del forraje verde hidropónico y germinado orgánico de maíz: Estudio de caso. Entelequia, $1(3), 5$.

Osorno, R. A. y González, L. M. (2012). Producción y calidad de la biomasa de Zea mays, Sorghum bicolor, Oriza sativa en alfombra forrajera hidropónica [Tesis de pregrado, Universidad Nacional Agraria]. Repositorio institucional. https://repositorio. una.edu.ni/1459/1/tnf01083.pdf
Paipa, L., Bernal, L., Conde, A., Quijano, N. y Bula, K. (2020). El forraje verde hidropónico: una alternativa sostenible en tiempos de cambio climático. Ámbito Investigativo, 5(2), 60-71. https://ciencia.lasalle.edu.co/cgi/ viewcontent.cgi?article $=1107 \&$ context $=a i$

Ramírez, C. y Soto, F. (2017). Efecto de la nutrición mineral sobre la producción de forraje verde hidropónico de maíz. Agronomía Costarricense, 41(2), 79-91. https://doi. org/10.15517/rac.v41i2.31301

Ruiz-Sesma, D. L., Lara-Lara, P. E., SierraVázquez, Á. C., Aguilar-Urquizo, E., MagañaMagaña, M. A. y Sanginés-García, J. R. (2006). Evaluación nutritiva y productiva de ovinos alimentados con heno de Hibiscus rosasinensis. Zootecnia Tropical, 24(4), 467-482. http://ve.scielo.org/scielo.php?script $=$ sci arttext\&pid=S0798-72692006000400006

Saguilán, P. C., Aguirre, H. A., MartínezMartínez, R. Gómez, A., Maldonado, M. A. y Ayala, M. A. (2020). Forraje verde hidropónico en dietas de cerdos en crecimiento en Pinotepa Nacional, Oaxaca. Revista Mexicana de Ciencias Agrícolas, 11(24), 247-253. https:// doi.org/10.29312/remexca.v0i24.2375

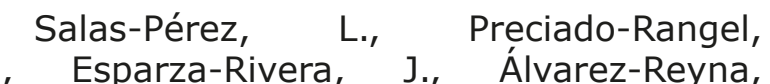
V. P., Palomo-Gil, A., Rodríguez-Dimas, N., y Márquez-Hernández, C. (2010). Rendimiento y calidad de forraje hidropónico producido bajo fertilización orgánica. Terra Latinoamericana, 28(4), 355-360. http:// www.scielo.org.mx/scielo.php? script $=$ sci arttext\&pid=S0187-57792010000400007

Sánchez, F., Moreno, E. C., Contreras, E. y Morales, J. (2013). Producción de forraje hidropónico de trigo y cebada y su efecto en la ganancia de peso de borregos. Revista Chapingo Serie horticultura, 19(4), 35-43. http://www.scielo.org.mx/ scielo.php?script $=$ sci_arttext\&pid $=$ S1027$152 \times 2013000400003$

Tarrillo, H. (2007). Forraje verde hidropónico, forraje de alta calidad, para la alimentación animal.

Trevizan, J. F. y Challapa, G. A. (2020). Comparación del rendimiento de forraje verde 
hidropónico con maíz lluteño y maíz comercial, utilizando cuatro calidades de agua. Arica, Chile. Idesia, 38(3), 113-122. http://dx.doi. org/10.4067/S0718-34292020000300113

Vargas-Rodríguez, C. F. (2008). Comparación productiva de forraje verde hidropónico de maíz, arroz y sorgo negro forrajero. Agronomía mesoamericana, 19(2), 233-240. https://www.redalyc.org/ pdf/437/43711425008.pdf

Conflicto de intereses

Los autores declaran no tener ningún conflicto de intereses. 